\title{
Musik bei der Kolposkopie reduziert die Angst
}

Viele Frauen haben Angst, wenn sie sich wegen eines auffälligen Zervixabstrichs einer Kolposkopie unterziehen müssen. Ein aktueller Cochrane-Review ging der Frage nach, welche Intervention ihnen die Prozedur vielleicht erträglicher machen kann.

$\mathrm{D}$ as Zervixscreening kann auf allen Stufen Angst hervorrufen. Das ist für die Betroffenen belastend und hat u.U. negative Konsequenzen. Frauen, die vor und während einer Kolposkopie viel Angst haben, erleben die Untersuchchung eher als schmerzhaft und unangenehm. Außerdem besteht die Gefahr, dass sie sich der weiteren Abklärung des auffälligen Abstrichs verweigern.

Das angstreduzierende Potenzial verschiedener Interventionen vor und während der Kolposkopie nahm jetzt ein Cochrane-Review anhand von sechs randomisierten kontrollierten Studien mit Daten von 886 Frauen unter die Lupe.

Als besonders erfolgversprechend erwies sich Musik bei der Kolposkopie. In einer Studie, die immerhin 220 Frauen einschloss, nahm der Angstpegel durch eine musikalische Untermalung der Untersuchung signifikant ab. Auch die berichteten Schmerzen gingen signifikant zurück. Das Aushändigen einer Informationsbroschüre vor der Untersuchung wirkte sich dagegen nicht signifikant auf den Angstpegel aus, doch waren positive Effekte auf Kenntnisstand sowie psychosexuelle Dysfunktion nachweisbar. Auch eine Beratung zusätzlich zu einer Informationsbroschüre (und einem Informationsvideo) machte die Frauen nicht weniger ängstlich. Eine als „quasi randomisiert" eingestufte Studie mit 81 Teilnehmerinnen ergab, dass Frauen signifikant weniger Angst haben, wenn sie die Un-

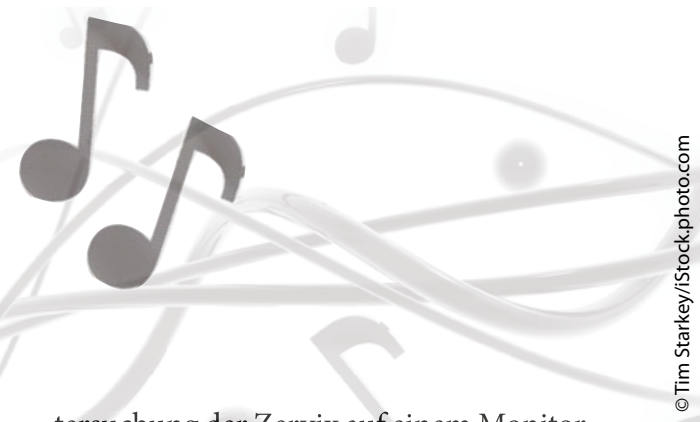

tersuchung der Zervix auf einem Monitor verfolgen können.

Fazit: Frauen, die bei der Kolposkopie Musik hören oder die Untersuchung auf einem Bildschirm sehen können, haben deutlich weniger Angst als andere. Die Autoren des Cochrane-Review sehen in der Musikuntermalung eine einfache und billige Möglichkeit, Ängste abzubauen und Schmerzen zu reduzieren. Sie regen an, die Frauen ihre eigene Musik zur Kolposkopie mitbringen zu lassen.

Ulrike Wepner

Galaal K et al. Interventions for reducing anxiety in women undergoing colposcopy. Cochrane Database Syst Rev. 2011 Dec 7;(12):CD006013.

\section{Mit Kaugummi \& Co. gegen Mundtrockenheit}

Bei Krebspatienten kann eine Bestrahlung im Kopf-Hals-Bereich, aber auch eine Chemotherapie zu unangenehmer Mundtrockenheit führen. Ein aktueller Cochrane-Review untersuchte, was topische Therapien hier bringen.

$\mathrm{M}$ undtrockenheit ist nicht nur unangenehm, sondern kann auch Schwierigkeiten beim Sprechen, Kauen und Schlucken bereiten. Zahnprobleme und negative Auswirkungen auf die Lebensqualität drohen. Für den CochraneReview gesichtet wurden 36 randomisierte kontrollierte Studien mit insgesamt

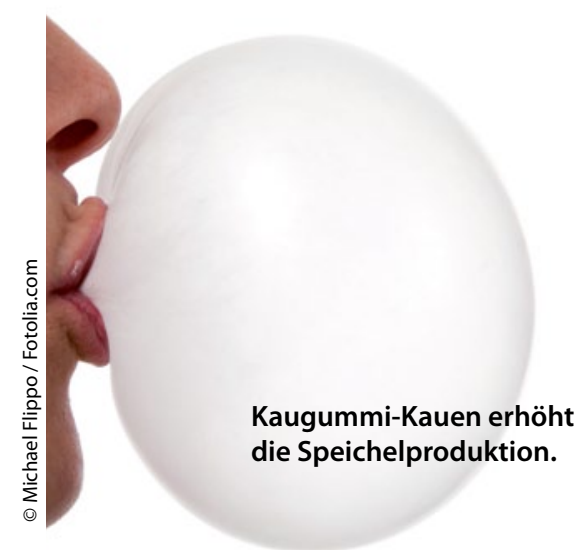

1.597 Teilnehmern, in denen topische Therapien zur Linderung von Mundtrockenheit unterschiedlicher Ursache zur Anwendung kamen. Auf dem Prüfstand standen verschiedene Speichelersatzmittel und Mittel zur Anregung des Speichelflusses.

Die Autoren kommen zu dem Schluss, dass keine topische Therapie wirklich die Symptome der Mundtrockenheit entscheidend lindern kann. Ein Spray mit oxygeniertem Glyceroltriester als Speichelersatz, der die Mundschleimhaut mit einem Lipidfilm versieht, erwies sich im Vergleich mit einem wässrigen Elektrolytspray als effektiver. Der Unterschied entsprach ungefähr einer mittleren Differenz von zwei Punkten auf einer visuellen Analogskala (VAS) von zehn Punkten zur Mundtrockenheit. Auch für muzinhaltige Sprays oder Lutschtabletten gibt es positive Daten. Integrierte Mundpflegesysteme (Zahnpasta plus Gel plus
Mundwasser) und Gel freisetzende Reservoirsysteme werden ebenfalls als vielversprechend eingestuft, allerdings fehlt bisher die Evidenz, um ihren Einsatz empfehlen zu können.

Mit Kaugummis lässt sich bei der Mehrzahl der Patienten, die noch eine Restaktivität der Speicheldrüsen aufweisen, eine Zunahme der Speichelproduktion erzielen. Sie werden von den Patienten meist gern verwendet. Es gibt jedoch keine Evidenz, dass sie mehr oder weniger effektiv sind als Speichelersatzmittel.

Fazit: Nach diesem Review fehlt bisher die Evidenz, um eine spezifische topische Therapie zur Bekämpfung von Mundtrockenheit wirklich empfehlen zu können. Größere Studien mit gutem Design wären erforderlich. Ein wichtiger Aspekt im Behandlungsalltag ist die Präferenz des $\mathrm{Pa}$ tienten. Auf den Einsatz säure- und zuckerhaltiger Produkte sollte verzichtet werden.

Ulrike Wepner

Furness $\mathrm{S}$ et al. Interventions for the management of dry mouth: topical therapies.

Cochrane Database Syst Rev. 2011 Dec 7;(12):CD008934.. 